\title{
Alfresco workout for optimal postpartum reclamation
}

\begin{abstract}
This article delineates an individual and public requirement for timely alfresco or open-air (outdoor) physical training postpartum for early and healthy recovery with minimal drawbacks. A global standard of a minimum daily exercise of 25 $30 \mathrm{~min}$ serious walking as an equivalent for after the first few days postpartum is recommended. In caesareans, however, serious outdoor physical activity should be delayed until after the first few weeks postpartum, depending on neonate and maternal physiological and health states. Setting simple and definitive global guidelines for resumed exercise postpartum in open-air conditions is an inevitable part of optimal public health programs in today's increasingly modernizing time.
\end{abstract}

Keywords: exercise, postpartum, maternal recovery, outdoor activity
Volume 3 Issue 4 - 2015

\author{
Akbar Nikkhah \\ Department of Animal Sciences, University of Zanjan, Iran \\ Correspondence: Akbar Nikkhah, Chief Highly Distinguished \\ Professor, Foremost Principal Highly Distinguished Elite- \\ Generating Scientist, Department of Animal Sciences, Faculty \\ of Agricultural Sciences, University of Zanjan, National Elite \\ Foundation, Iran, Email anikkha@yahoo.com
}

Received: August 20, 2015 | Published: November 12, 2015

\section{Public description of science and philosophy}

Discussion on the necessity of physical activity during pregnancy has long been on. Also, efforts have been made to facilitate postpartum exercise timely. However, limited attention has been given towards the essentiality of adequate outdoor physical work early postpartum for healthy recovery involving timely uterus involution and optimal ovaries and reproductive health and efficiency. However, it must be noted that inter-mother differences do exist, and thus, a major medical challenge has been to develop sound strategies that can be applied on large national and global scales. But, what sounds certain is the fact that the possibility does occur for a mother after a normal natural delivery to resume outdoor fresh-air exercise as early as few days (e.g., 4-5days) postpartum.

The main innovative aspect of this article relates to its outdoor fresh-air nature of exercise, exposing the fresh mother to natural air conditions. However, the open-air exercise must be initiated gradually and increased little by little, preferably on a daily or every other daily rhythm. The outdoor physical work mostly represents gentle walking the rate and duration of which may be enhanced over time. This can be converted to gentle running later on to become more serious afterwards. For instance, modest long-distance running may possibly easily start by about $6-8$ weeks postpartum or likely earlier. This exercise program works best in mothers that were conducting regular and serious exercise during the entire pregnancy until just before parturition. Aerobic outdoor activities (e.g., walking, climbing, running) are considered optimal for both light and heavy pregnancies the timely resumption of which after delivery results in improved maternal-neonatal health and resistance against a variety of pathogens.

The success of early serious exercise programs postpartum highly depends on maternal weight management as well. ${ }^{1,2}$ The regularity and rhythmicity of adequate physical work long before and indeed during pregnancy provides safety against unwanted obesity, including dramatically harmful central adiposity. ${ }^{3,4}$ As such, such prolonged exercise programs must be conducted in parallel with optimal eating and resting regimens. ${ }^{1-5}$ Frequent small meals during day and no major large meal overnight are amongst the essential nutritional elements for successful and health-improving individual and public exercise strategies. ${ }^{6-11}$ Preventing diabetes and combating obesity and related metabolic abnormalities can only be aimed for with optimal timing and intensity of both eating and exercise. ${ }^{6,9,10}$ Recently, a philosophy has been developed to harmonize eating and exercising intensity on a circadian or daily basis not longer. ${ }^{3}$ This implies that conducting regular daily exercise with relative intensity is safer and more healthful than overly serious exercise conducted merely once weekly or in any comparable long interval. In a nutshell, it is the regularity, rhythmicity, frequent, and the daily nature of the exercise that matters most in optimizing human physiology and health. This applies to pregnant and lactating mothers very appropriately, since metabolic pressures on the body are dramatically intense, and thus, more relief may be obtained through intense-enough well-disciplined exercise programs. ${ }^{12}$

\section{Implication}

Outdoor physical work for aerobic refreshing of reproductive and other peripheral tissue can be a safe strategy to hasten healthy maternal recovery and improve lactation for optimal neonate and child wellbeing. However, conducting the fresh-air exercise programs early enough and successfully requires prolonged programming of aerobic physical activity long before and during the entire gestation. Eating and resting regimens must also be optimized regularly in parallel with open exercise, preferably on a short-interval basis of daily or every other daily, for instance.

\section{Acknowledgements}

Thanks to the ministry of Science Research and Technology, National Elite Foundation, and University of Zanjan for supporting the author's global initiatives and programs of optimizing science edification in the third millennium.

\section{Conflict of interest}

The author declares no conflict of interest.

\section{References}

1. Nikkhah A. Harmonizing eating and exercise circadian rhythms for optimal glucose-insulin and vascular physiology. Int J Diabetol Vasc Dis Res. 2015;3(3):87-88. 
2. Nikkhah A. Secure weight management via fitting circadian patterns of physical activity, resting and eating. Adv Obes Weigh Manag Cont. 2015;2(4):00023.

3. Nikkhah A. Circadian fitting of exercise and eating patterns: The secret of healthy life. J Bioprocess Biotech. 2015;5:e129.

4. Nikkhah A. Breast health progress through exercise-driven lactation: A pragmatic bioprocess to prevent cancer. J Bioprocess Biotechniq. 2015;5:3.

5. Nikkhah A. Optimizing gestation and early life physiology through timing of energy turnover: Bioprocessing of human life. J Bioprocess Biotechniq. 2015;5:e125.

6. Nikkhah A. Eating timing and diabetes. Int J Diabetol Vas Dis Res. 2014;2(2):1.
7. Nikkhah A. Untimely intake as a postmodern public health bioterrorism J J Bioterror Biodef. 2015;7:e118.

8. Nikkhah A. Optimizing health management through circadian feed intake rhythms: A prognostic SciTech. J Vet Sci Technol. 2015;6:e115.

9. Nikkhah A. Avoid large night meals to stay fit. J Obes Weight Loss Ther. 2015;4:e115.

10. Nikkhah A. When to eat to beat obesity and diabetes? Journal of Diabetes and Metabolism. 2014;5:7.

11. Nikkhah A. Circadian timing of hunger to avert obesity: An environmental evolutionary science. J J Environ Sci. 2015;1(1):005.

12. Nikkhah A. Social Health Betterment via Optimizing Embryonic and Foetal Omics: An Obesity Perspective. Adv Obes Weight Manag Control. 2015;3(1):00041 